\title{
MTA1 downregulation inhibits malignant potential in a small cell lung cancer cell line
}

\author{
HONGSHENG XUE ${ }^{1,2^{*}}$, HAIJUAN WANG ${ }^{1 *}$, JIAN LIU $^{1}$, HUAN LIU $^{1}$, CHUNXIAO LI $^{1}$, \\ LI HAN $^{2}$, CHEN LIN $^{1}$, QIMIN ZHAN ${ }^{1}$, ZHILONG ZHAO $^{2}$ and HAILI QIAN ${ }^{1}$ \\ ${ }^{1}$ State Key Laboratory of Molecular Oncology, Cancer Institute and Hospital, \\ Chinese Academy of Medical Sciences, Beijing 100021; ${ }^{2}$ Department of Thoracic Surgery, \\ The Affiliated Zhongshan Hospital of Dalian University, Dalian 116001, P.R. China
}

Received July 31, 2014; Accepted October 30, 2014

DOI: 10.3892/or.2014.3671

\begin{abstract}
As a component of the nuclear remodeling and deacetylation complex (NuRD complex), metastasis-associated gene 1 (MTA1) has been reported to play a key role in cancer malignancy. However, whether MTA1 functions in small cell lung cancer (SCLC) malignant behavior and whether it is feasible to be used as a therapeutic target have not been evaluated. The present study aimed to investigate the effects of MTA1 downregulation on SCLC malignancy. First we demonstrated the overexpression of MTA1 in SCLC specimens. After knocking down the MTA1 level by specific siRNA sequence, the biological consequences on proliferation, migration, invasion and apoptosis were evaluated. The results showed that MTA1 silencing had potent suppressive effects on SCLC proliferation, migration and invasion. Apoptosis but not cell cycle arrest was induced in the MTA1-silenced SCLC cells. In summary, MTA1 plays a critical role in regulating the malignant behaviors of SCLC. Depleting MTA1 level may be an effective strategy by which to suppress SCLC growth and metastasis in future biotherapeutic attempts.
\end{abstract}

\section{Introduction}

Lung cancer is one of the most deadly diseases and ranks first as the cause for cancer-related mortality (1). Small cell lung cancer (SCLC), accounting for $\sim 15 \%$ of all lung cancer cases,

Correspondence to: Professor Zhilong Zhao, Department of Thoracic Surgery, The Affiliated Zhongshan Hospital of Dalian University, Dalian 116001, P.R. China

E-mail: zhilong509@126.com

Dr Haili Qian, State Key Laboratory of Molecular Oncology, Cancer Institute and Hospital, Chinese Academy of Medical Sciences, Beijing 100021, P.R. China

E-mail: qianhaili001@163.com

*Contributed equally

Key words: metastasis-associated gene 1, small cell lung cancer, metastasis, invasion is a highly aggressive subtype with neuroendocrine properties (2). Currently, for SCLC, there is no effective treatment strategy either through surgery or chemotherapy (3), attributed to its early dissemination and fast development of drug resistance after initiation of chemotherapy (4). The 5-year survival of SCLC patients is as low as $15 \%$ or less even after aggressive treatment (5). In light of targeted therapeutic approaches which have shown advantages over traditional strategies, new therapeutic targets must be explored based on the ongoing understanding of molecular mechanisms in lung cancer development and progression.

Cancer development and progression are characterized by deregulated gene expression networks which drive the proliferation and metastasis of cancer cells (6). Among the deregulated cancer-related genes, metastasis-associated gene 1 (MTA1) is one of the key players involved in certain steps of cancer development (7). MTA1 is a component of the nuclear remodeling and deacetylation complex (NuRD complex) that affects gene expression ubiquitously (8). MTA1 has been reported to be overexpressed in a series of malignant diseases, including breast cancer (9), esophageal squamous cell carcinoma (10), oral squamous cell carcinoma (11), colon cancer (12) and nonsmall cell lung cancer (NSCLC) (13). Moreover, it has been shown to exert cancer progression in a number of cancer types, such as melanoma, esophageal squamous cell carcinoma and colon cancer, showing promise as a molecular target in cancer therapy strategy development (14). However, whether MTA1 also plays a key role in SCLC malignant behavior or whether it also bears promise in SCLC treatment needs to be ascertained before further potential MTA1-targeted therapeutics can be evaluated in this subtype of cancer.

Thus, in the present study, we focused on the expression status of MTA1 in SCLC and the in vitro and in vivo biological effects of MTA1 silencing to evaluate its potential as a therapeutic target in SCLC.

\section{Materials and methods}

Cell lines and cell culture. NCI-H446 (human SCLC), A549 and H1650 (human NSCLC) cell lines were maintained in our laboratory. The cells were cultured in RPMI-1640 supplemented with $10 \%$ newborn calf serum, penicillin $(100 \mathrm{U} / \mathrm{ml})$, 
and streptomycin $(100 \mathrm{mg} / \mathrm{ml})$. The cells were incubated at $37^{\circ} \mathrm{C}$ in a humidified atmosphere with $5 \% \mathrm{CO}_{2}$.

Small interfering RNA transfection. The MTA1 small interfering RNA (siRNA) and negative control siRNA were purchased from Shanghai GenePharma Co., Ltd. (Shanghai, China). The sequence of the MTA1 siRNA was 5'-GACCCTG CTGGCAGATAAA-3'. The siRNAs were dissolved in sterilized and RNase-free water to the final concentration of $20 \mu \mathrm{M}$. Lipofectamine 2000 (11668-019; Life Technologies, Grand Island, NY, USA) was used to transfect the siRNAs into cells according to the manufacturer's instructions. Briefly, NCI-H446 cells were seeded in 6-well plates at a density of $3 \times 10^{5}$ cells/well in RPMI-1640 medium in $37^{\circ} \mathrm{C}$ incubator until reaching $70 \%$ confluency. After washing the cells with phosphate-buffered solution (PBS) and serum-free RPMI-1640 medium, $5 \mu 1$ siRNA was mixed with $5 \mu 1$ Lipofectamine 2000 in $1.5 \mathrm{ml}$ serum-free RPMI-1640 medium at room temperature for $20 \mathrm{~min}$. Then the siRNALipofectamine 2000 complex mixture was added into the wells and was replaced with RPMI-1640 medium containing $10 \%$ fetal bovine serum after $6 \mathrm{~h}$. The cells were continuously incubated for $48 \mathrm{~h}$ before they were harvested for silencing efficiency and biological assays.

Polymerase chain reaction (PCR). The polymerase chain reaction (PCR) was performed as previously described (13). Briefly, total RNA was extracted from the variously treated cells using TRIzol reagent (15596-026; Life Technologies). First Strand cDNA was synthesized from mRNA using vigoScript First Strand cDNA Synthesis kit (11904-018; Life Technologies). The following primers were used to amplify the MTA1 fragment: forward primer, 5'-CAGCTACGAGCAGC ACAACG-3' and reverse primer, 5'-TGTCCGTGGTTTGCC AGA-3'. Glyceraldehyde phosphate dehydrogenase (GAPDH) was amplified as an internal control using forward primer, 5'-GGTGGTCTCCTCTGACTTCAACA-3' and reverse primer, 5'-GTTGCTGTAGCCAAATTCGTTGT-3'. PCR was performed using an ABI Veriti ${ }^{\circledR}$ 96-Well Thermal Cycler (4375786; Life Technologies). The cycling conditions were as follows: initial denaturation at $94^{\circ} \mathrm{C}$ for $60 \mathrm{sec}$, followed by 28 cycles at $94^{\circ} \mathrm{C}$ for $60 \mathrm{sec}, 60^{\circ} \mathrm{C}$ for $60 \mathrm{sec}$ and $72^{\circ} \mathrm{C}$ for $30 \mathrm{sec}$. The experiment was repeated three times for each test.

Western blot analysis. Western blot analysis was performed as described in a previous publication (15). The protein lysis was subject to $10 \%$ sodium dodecyl sulfate-polyacrylamide gel electrophoresis (SDS-PAGE) and then electrotransferred to polyvinylidene fluoride (PVDF) membranes. After blocking with 5\% fat-free milk in Tris-buffered saline Tween-20, the PVDF membranes were incubated with the anti-MTA1 polyclonal antibody (ab71153; Abcam, Cambridge, MA, USA), anti- $\beta$-actin monoclonal antibody (A5316; Sigma-Aldrich, St. Louis, MO, USA), anti-p53 polyclonal antibody (sc-126; Santa Cruz Biotechnology, Inc., Dallas, TX, USA), anti-E-cadherin polyclonal antibody (ab11512; Abcam), anti-PARP polyclonal antibody (\#9542; Cell Signaling Technology, Inc., Danvers, MA, USA), anti-PUMA polyclonal antibody (\#4976; Cell Signaling Technology), anti-cyclin D1 or anti-cyclin E1 polyclonal antibody (bs501741, bs501085; Bioworld Technology,
Nanjing, China), overnight at $4^{\circ} \mathrm{C}$, followed by horseradish peroxidase-conjugated secondary antibody $(1: 5,000)$ incubation for $1 \mathrm{~h}$ at room temperature. Signals were detected using LAS 4000 Imaging system (Fijifilm, Tokyo, Japan). The experiment was repeated twice.

Cell proliferation assay. 3-(4,5-Dimethylthiazol-2-yl)5-(3-carboxymethoxy phenyl)-2-(4-sulfophenyl)-2H-tetrazolium (MTS) assay was used to detect cell proliferation. Briefly, cells were seeded in complete growth medium in 96-well plates at a density of $3 \times 10^{3}$ cells/well. After incubating for 1, 2, 3, 4 and 5 days, the medium in each well was replaced with $100 \mu \mathrm{l}$ fresh medium and $10 \mu \mathrm{l}$ of aqueous solution of cell proliferation MTS reagent and incubated at $37^{\circ} \mathrm{C}$ for $2 \mathrm{~h}$. The OD values of each well were measured with a microplate reader at $\lambda=490 \mathrm{~nm}$.

Colony formation assay. The cells after being transfected with siRNA were incubated for $48 \mathrm{~h}$ and then harvested and resuspended in complete medium at a concentration of $1 \times 10^{3}$ cells/ $\mathrm{ml}$. Then, $0.5 \mathrm{ml}$ of the cell suspensions was added into the 6-well plates, and $2.5 \mathrm{ml}$ complete medium was added. After 10 days, the plates were washed with PBS twice and stained with $0.2 \%$ crystal violet dye for $10 \mathrm{~min}$. Finally, the cells were photographed and analyzed under an imaging system (G:BOX, Syngene, Cambridge, UK). The detection was repeated twice with duplicate measurements in each experiment.

Adhesion assay. A 96-well plate was precoated with $50 \mu 1$ Matrigel (cat. no 356234; BD Biosciences, Bedford, MA, USA, $10 \mathrm{mg} / \mathrm{ml}$ ) in advance. Then the three cell lines were harvested and reseeded into 96-well plates in complete growth medium at a density of $3 \times 10^{4}$ cells/well. Unattached cells were washed three times with PBS gently at different time-points, and the cells remaining on the wells were quantified using the MTS assay. The percentages of adhered cells were calculated relative to the cells in the unwashed control wells. Serial dilutions were conducted to construct a standard curve to convert absorbance at $490 \mathrm{~nm}$ to cell number.

Transwell invasion assay. The invasion assay was performed using Transwell chambers (\#3422; Corning Life Sciences, Tewksbury, MA, USA) with $50 \mu 1$ Matrigel-precoated (cat. 356234; BD Biosciences, San Jose, CA, USA, $10 \mathrm{mg} / \mathrm{ml}$ ) polycarbonate membrane as described in a previous report (15). Briefly, the three groups of cells were collected and resuspended in serum-free RPMI-1640 medium at a concentration of $1.5 \times 10^{5}$ cells $/ \mathrm{ml}$, then the cell suspensions were added into the top chambers $(200 \mu \mathrm{l} / \mathrm{well})$ and the bottom chambers were filled with complete medium ( $500 \mu \mathrm{l} / \mathrm{well})$, followed by a $24-\mathrm{h}$ incubation at $37^{\circ} \mathrm{C}$. The cells that did not penetrate the polycarbonate membrane were swabbed using a cotton bud. The cells that transmembraned through and adhered to the bottom of the polycarbonate membrane were stained with $0.2 \%$ crystal violet dye for $10 \mathrm{~min}$, and the number of invaded cells was counted. The experiment was repeated twice with duplicate measurements in each experiment. For each well, five fields were chosen (the ones in the middle, at 3, 6,9 and 12 time clock positions) to count the total cell numbers. Data from three parallel wells were averaged and compared between the groups. 

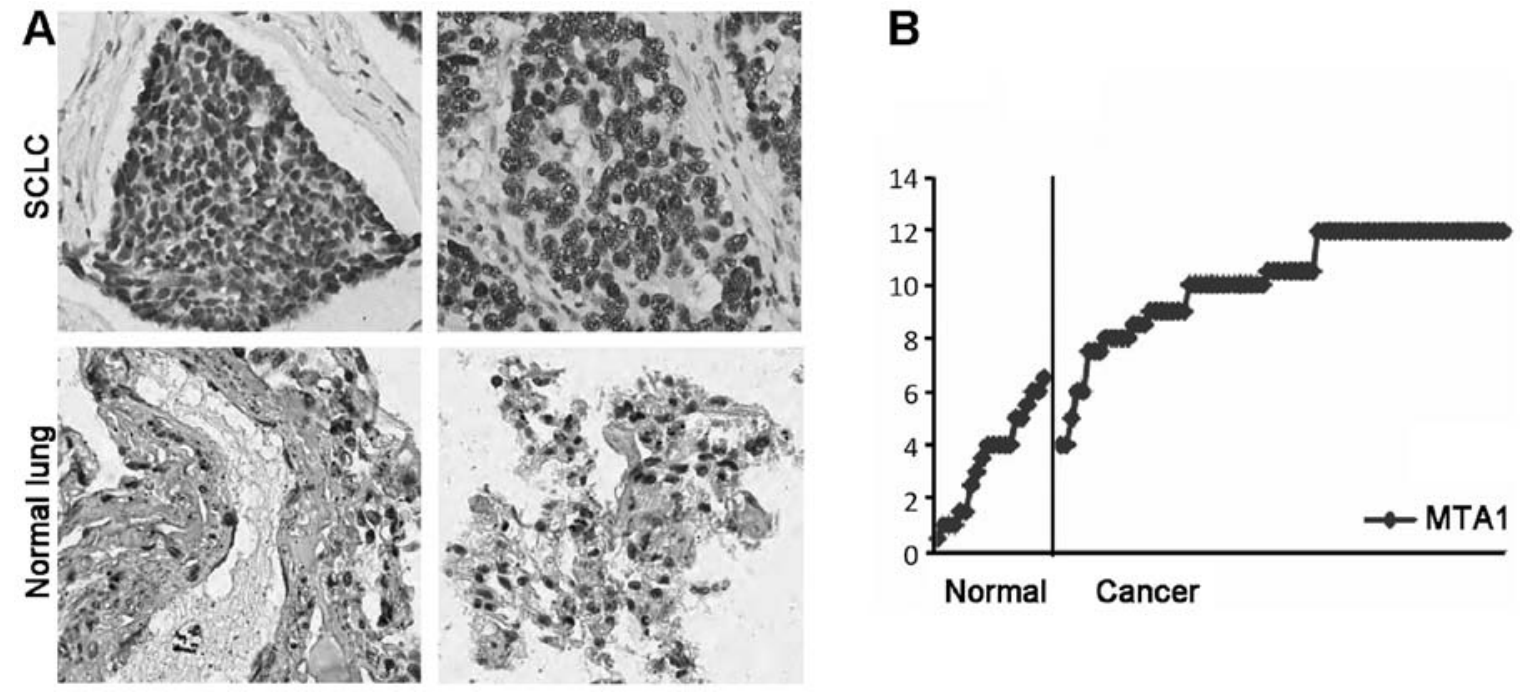

Figure 1. MTA1 is highly expressed in SCLC specimens compared with normal lung tissues. (A) Representative images of immunohistochemistry in the SCLC tissue microarrays. The staining of MTA1 in SCLC was significantly stronger than normal lung tissues (magnification, x200). (B) The immunohistochemistry results showed that MTA1 was overexpressed in SCLC ( $\mathrm{P}<0.05$ vs. normal lung tissue). SCLC, small cell lung cancer; MTA1, metastasis-associated gene 1.

Wound healing assay. Wound healing assay was performed as previously described (16). Briefly, the same amount of cells was seeded in a 6-well plate and incubated until the cells grew to $80 \%$ confluencey. A sterile pipette tip was used to scratch a line on the monolayer cells with the same width. The detached cells were washed off with PBS and the remaining cells were cultured in complete medium. Specific points on the scratched lines were photographed at 0,12 and $24 \mathrm{~h}$ after scratching. The healing of the wounds was presented by the distance between the frontline of cells moving towards the scratched area. The experiment was repeated in triplicate.

Annexin V FITC assay and cell cycle analysis by flow cytometry. The variously transfected cells were incubated for $48 \mathrm{~h}$ and then trypsinized. For theAnnexin V FITC assay, the cells were washed with PBS twice and stained with Annexin V-FITC and PI (cat. 556547; BD Biosciences) for $15 \mathrm{~min}$ at room temperature and analyzed by flow cytometry for cells undergoing apoptosis. For cell cycle analysis, cells were washed with PBS twice and then fixed with cold $70 \%$ ethanol at $-20^{\circ} \mathrm{C}$ overnight. The cells were treated with RNase A for 30 min at $37^{\circ} \mathrm{C}$, stained with propidium iodide (PI) solution for $30 \mathrm{~min}$ at $4^{\circ} \mathrm{C}$ and then measured by flow cytometry (cat. 643183; BD Biosciences). ModFit LT software (VMFLTWIN4, Verity Software House Inc., Topsham, ME, USA) was used for data acquisition and analysis.

Immunohistochemical staining of the tissue microarray (TMA). Paraffin-embedded small cell lung cancer TMAs (cat. no. LC10010a) were purchased from Alenabio Biotech Ltd. (Xi'an, China). The tissues on the TMA contained duplicate samples from 40 tumors and 10 normal lung tissues. Tumors on the TMA were categorized based on the TNM classification of tumors, as shown in Table I, and were verified by adjacent H\&E staining sections by two independent pathologists.

The tissue microarrays were deparaffined and antigenretrieved routinely. Immunohistochemical staining was performed as previously described (17). Briefly, the slides of the TMAs were washed with PBS, quenched for $15 \mathrm{~min}$ in $3 \%$ $\mathrm{H}_{2} \mathrm{O}_{2}$, and blocked with $2 \%$ normal goat serum for $30 \mathrm{~min}$ at room temperature before the anti-MTA1 polyclonal antibody was applied overnight at $4^{\circ} \mathrm{C}$. Then the slides were incubated with the biotinylated secondary antibody followed by incubation with HRP-labeled streptavidin. 3,3'-Diaminobenzidine hydrochloride (DAB) was used for staining. Then slides were counterstained with hematoxylin before being mounted and examined by light microscopy.

MTA1 protein levels were assessed in the TMAs. The staining intensity scores were as follows (18): 0 (negative), 1 (weak), 2 (moderate), and 3 (strong). The percentage of MTA1positive cells was also score based on four categories: 0 was scored for $0-10 \%, 1$ for $>10-25 \%, 2$ for $>25-50 \%, 3$ for $>50-75 \%$, and 4 for $>75-100 \%$. Staining was scored as the product of the staining intensity and percentage scores.

Statistical analysis. Statistically significant differences between groups were determined using an unpaired Student's t-test. Statistical significance was defined at $\mathrm{P}<0.05$.

\section{Results}

MTAl is highly expressed in SCLC specimens compared with normal lung tissues. In order to ascertain whether MTA1 plays a role in SCLC development and progression, we assessed the expression of MTA1 in a set of tissue microarrays containing a series of SCLC lung cancer tissues. We analyzed the levels of MTA1 by immunohistochemistry in the tissue microarrays (TMAs), including 40 patients with small cell lung cancer and 10 cases of normal lung tissue (Table I). The median age of the patients when they received surgical treatment was 52.675 years (range, 32-76). MTA1 was scored according to the staining intensity and percentage of positive cells in an entire cancer cell population. As shown in Fig. 1A and B, the SCLC tissue exhibited a marked increase in MTA1 expression compared with the normal lung tissues. 

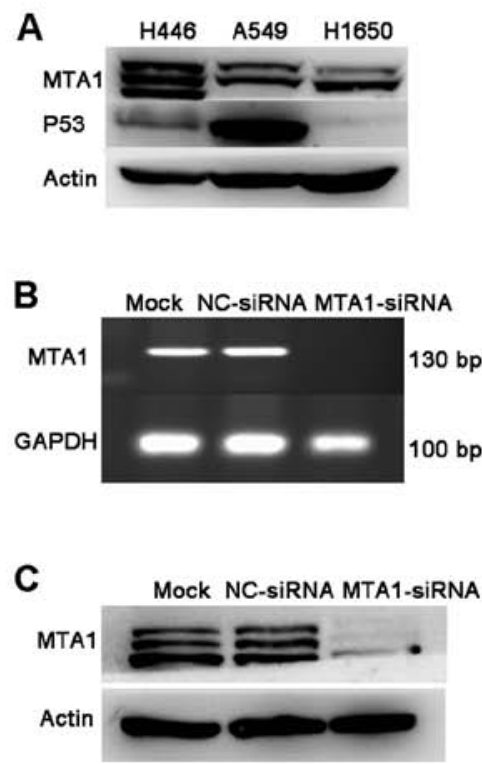

$\mathbf{E}$
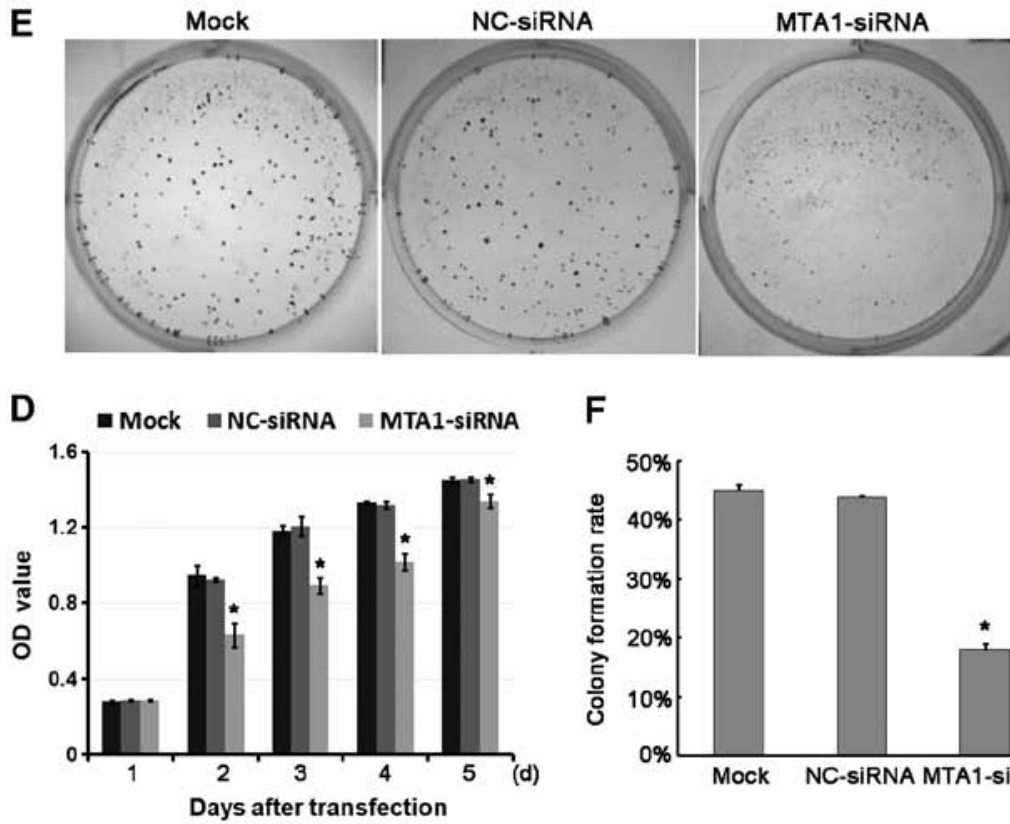

$\mathbf{F}$

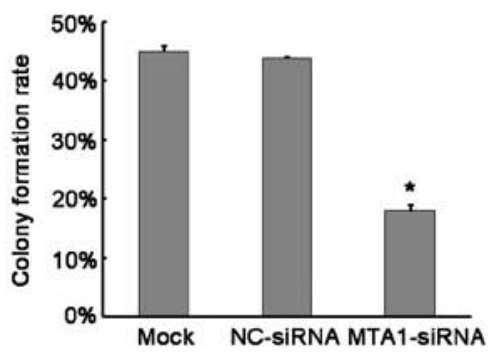

Figure 2. MTA1 downregulation suppresses the proliferation of the NCI-H446 cell line. (A) Expression of MTA1 in NCI-H446, A549 and H1650 cell lines. MTA1 was highly expressed in the SCLC cell line NCI-H446. (B and C) The results showed that the MTA1 siRNA specifically silenced the MTA1 mRNA and protein expression in the NCI-H446 cells. (D) The NCI-H446 cell line was treated with MTA1 siRNA for $24 \mathrm{~h}$. The MTS assay was used to determine the cell proliferation every $24 \mathrm{~h}$ for five days ("P $<0.05$, MTA1 siRNA vs. NC siRNA). (E) Colony formation assays were performed with mock, NC-siRNA and MTA1-siRNA cells, The panels show the images of the colonies stained with crystal violet. (F) Quantitative analysis of the percentage of colony formation of the NCI-H446 cells ( $\mathrm{P}<0.05$, MTA1 siRNA vs. NC siRNA).

Table I. Patient characteristics.

\begin{tabular}{lcc}
\hline Patient characteristics & No. of cases & $\%$ \\
\hline Gender (F/M) & $9 / 31$ & $22.5 / 77.5$ \\
Stage & & \\
I & 10 & 25.0 \\
II & 21 & 52.5 \\
III & 9 & 22.5 \\
Tumor & & \\
T1/T2 & 36 & 90 \\
T3/T4 & 4 & 10 \\
N0 & 12 & 30 \\
N1/N2 & 28 & 70 \\
Normal lung tissue & 10 & \\
\hline
\end{tabular}

MTA1 downregulation suppresses proliferation of the lung cancer NCI-H446 cell line. To ascertain whether MTA1 plays a positive role in SCLC proliferation, we used RNAi technique to knock down MTA1 expression in the SCLC cell lines and performed a proliferation assay. First, we screened a series of lung cancer cell lines for their MTA1 protein levels (Fig. 2A). We used NCI-H446 cells which express a high level of MTA1 as the model with which to evaluate the MTA1 function. After confirming the siRNA silencing efficiency (Fig. 2B and C), we compared the proliferation of the NCI-H446 cells between the MTA1-siRNA- and NC-siRNA-treated groups. We found that MTA1 siRNA exerted significant inhibition on cell growth (Fig. 2D). The colony formation ability was also suppressed by
MTA1 siRNA (Fig. 2E and F). Therefore, downregulation of MTA1 contributes to the tumor growth of SCLC.

Downregulation of MTA1 expression impairs the adhesion and invasive abilities of the SCLC cell line. MTA1 has been suggested to be involved in the process of metastasis. To ascertain whether MTA1 has an impact on SCLC cancer metastasis, we evaluated the adhesion and invasion assays in NCI-H446 cells. First, we determine the adhesion ability of NCI-H446 after MTA1 silencing. There was no significant difference in the adhesion ability between the mock group and the NC-siRNA-transfected group. However, the adhesion ability was greatly impaired in the MTA1-siRNA-treated NCI-H446 cells (Fig. 3A). Next, we explored the invasive potential of the NCI-H446 cells by the Transwell invasion model after MTA1siRNA and NC-siRNA transfection. We demonstrated that the MTA1-siRNA cells had significantly reduced numbers of invaded cells $(69.5 \pm 3.5$ vs. $424.5 \pm 11.5$ in the control) (Fig. 3B and $\mathrm{C}$ ). Adhesion and invasion are two key steps during tumor cell metastasis. To further confirm that MTA1 regulates SCLC metastasis at the molecular level, we detected alteration in the adhesion-associated protein E-cadherin by MTA1 manipulation. As shown in Fig. 3D, E-cadherin was much lower than that in the control group. These results suggest that MTA1 has a positive role in promoting SCLC metastasis.

MTA1 silencing reduces SCLC cell motility in the wound healing assay. Next, we performed a wound healing assay in the NCI-H446 cells to further understand the role of MTA1 in the migration potential of SCLC cells. The results demonstrated that the wound healing ability of the MTA1-siRNA group was significantly reduced when compared to that of the 


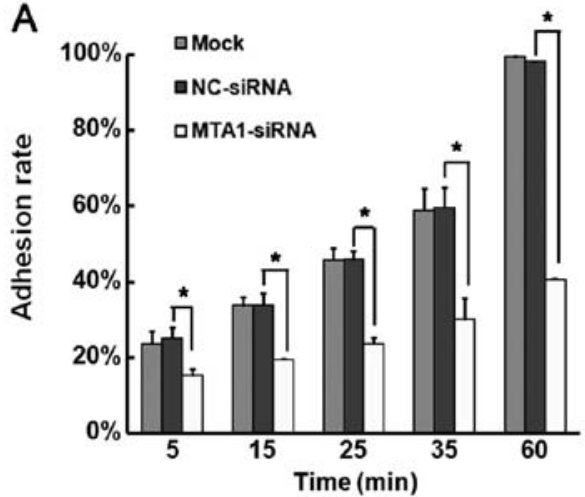

B

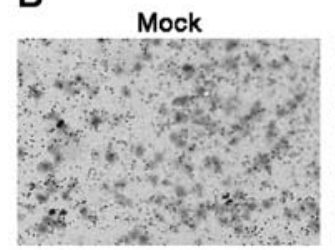

NC-siRNA

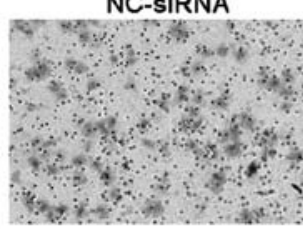

MTA1-siRNA

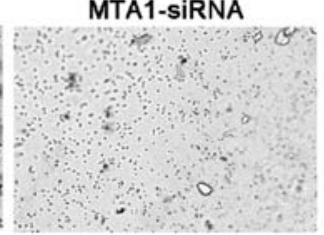

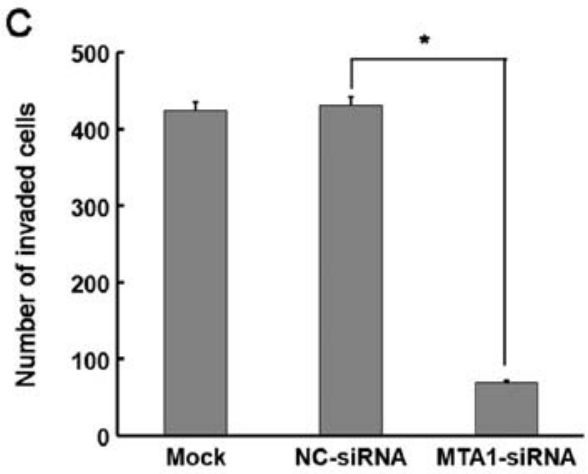

D

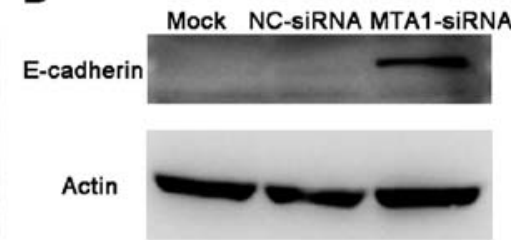

Figure 3. Downregulation of MTA1 expression impairs the adhesion and invasion abilities in the SCLC cell line. (A) Adhesion assays were performed in the mock, NC-siRNA and MTA1-siRNA cells. The adhesion of MTA1-siRNA cells was significantly reduced ("P<0.05, MTA1 siRNA vs. NC siRNA). (B) Images of the Transwell invasion assay in the mock, NC-siRNA and MTA1-siRNA cells. (C) Quantitative analysis of the number of invasive cells in the three cell groups. The number of invasive MTA1-siRNA cells was significantly reduced ("P $<0.05$, MTA1 siRNA vs. NC siRNA). (D) Western blot analysis showed that downregulation of MTA1 recruited the expression of E-cadherin.

A
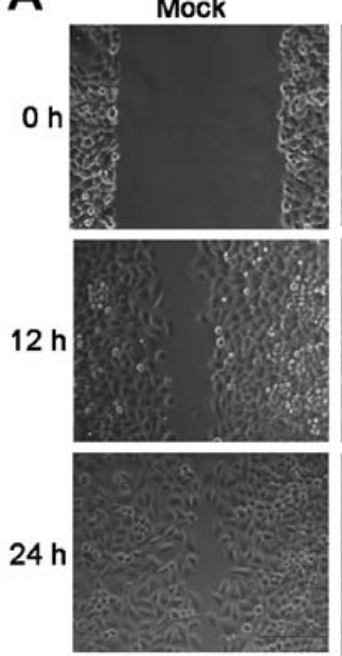

NC-siRNA
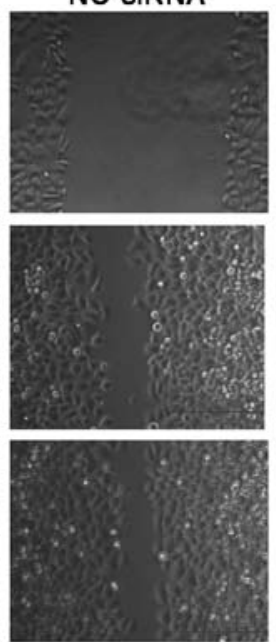

MTA1-SiRNA
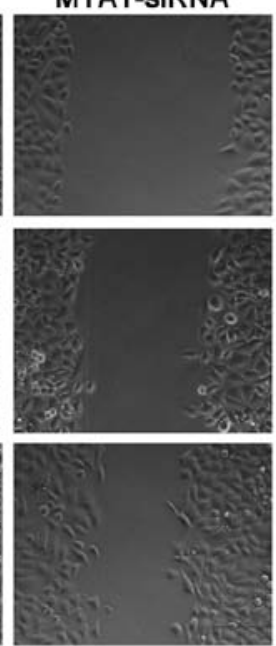

B

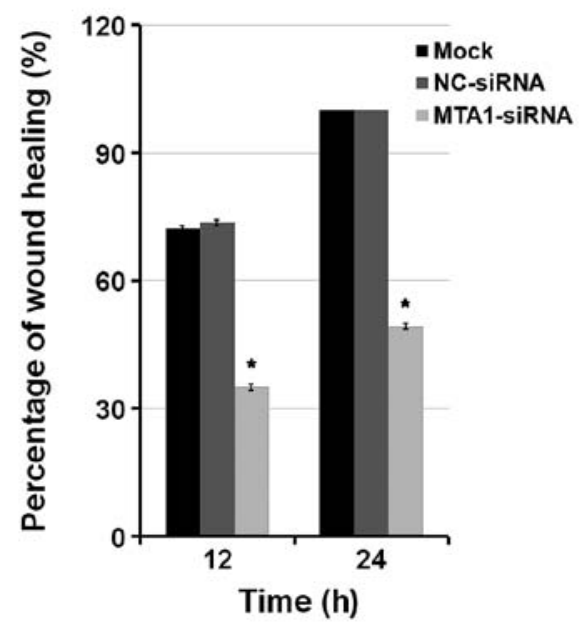

Figure 4. MTA1 silencing reduces SCLC cell motility in the wound healing assay. (A) Representative images of the mock, NC-siRNA and MTA1-siRNA cells in the wound healing assay (magnification, x200). (B) Quantitative analysis of the migration potential of NCI-H446 cells. The MTA1-siRNA cell wound healing was significantly reduced when compared with the negative control cells $\left({ }^{*} \mathrm{P}<0.05\right)$.

mock group and negative control group at $24 \mathrm{~h}$ after scratching. We measured the distance between the migrating frontlines and calculated the speed of wound healing. We found that the MTA1-siRNA-transfected group lagged behind the mock and control group in the healing process (Fig. 4). These data demonstrated that MTA1 was effective in promoting SCLC migration which was significantly inhibited by MTA1 silencing.

MTAl knockdown affects the apoptosis but not the cell cycle in the SCLC cell line. In order to explore whether MTA1 affects apoptosis in SCLC cell lines, we performed Annexin V FITC assay by flow cytometry. Data showed that MTA1 knockdown resulted in increased percentage of cells in both early and particularly late apoptosis, compared to the mock and negative groups (Fig. 5A). We further detected an alteration in apoptosisrelated proteins PARP and PUMA following MTA1 silencing. Results showed that MTA1-siRNA knockdown caused PUMA upregulation and PARP cleavage. We did not observe a significant change in the expression of cyclin E1 and cyclin D1, as consistent with the cell cycle assay by flow cytometry (Fig. 5B). 

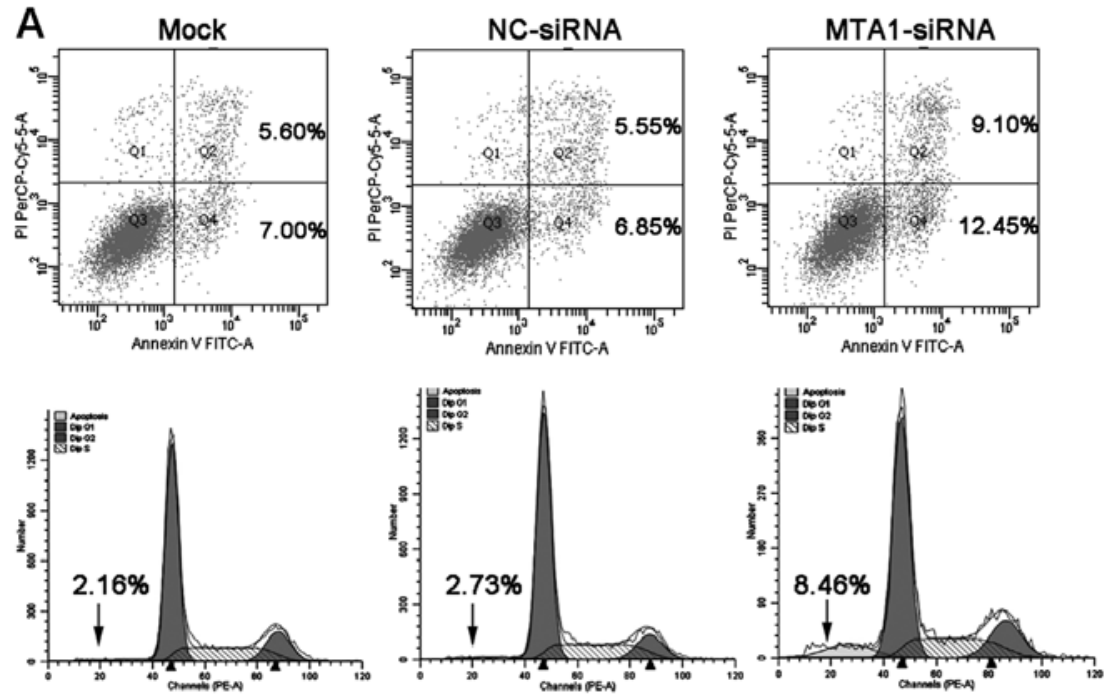

B

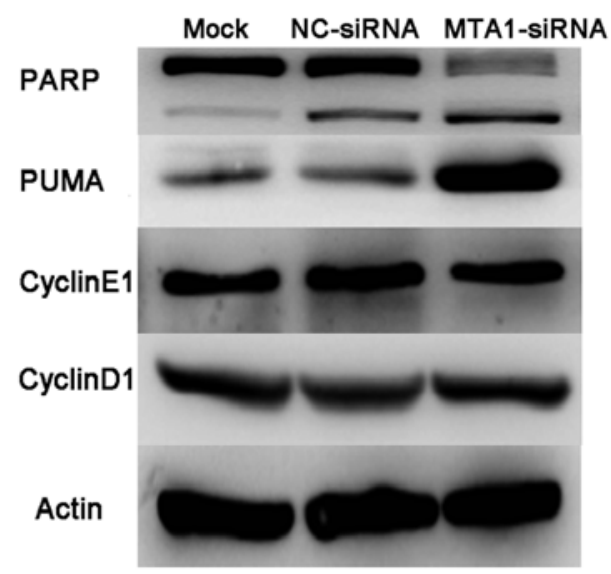

Figure 5. MTA1 knockdown increases apoptosis but does not affect the cell cycle in SCLC cells. (A) Annexin V FITC staining by flow cytometry revealed that the apoptosis of the NCI-H446 cells transfected with MTA1-siRNA was significantly increased while the cell cycle was not affected ${ }^{*} \mathrm{P}<0.05$, MTA1 siRNA vs. NC siRNA). (B) Western blot analysis showed that downregulation of MTA1 significantly increased the expression of PARP cleavage and PUMA, but did not significantly change levels of cyclin E1 and cyclin D1.

\section{Discussion}

Despite decades of biological and clinical research, there still remains a lack of consensus regarding the therapeutic protocol of patients with small cell lung cancer (SCLC). An understanding of the detailed molecular mechanisms are urgently needed, and a series of molecules have been linked to SCLC progression (19).

MTA1 is one of the molecules involved in carcinogenesis and cancer progression that has been discovered in the last few years. It was originally isolated from highly metastatic rat mammary adenocarcinoma cell lines by differential cDNA library screening (20). In human cancer, it was first reported to be significantly correlated with the malignant properties of gastrointestinal cancers in 1997 (21). Subsequent studies confirmed a widespread upregulation of MTA1 in various human tumors (22), contributing to multiple malignant properties of cancer, particularly invasion and metastasis.

The first evidence showing that MTA1 is upregulated in advanced lung cancer came in 2002 by Sasaki et al (23) via MTA1 mRNA detection in 74 non-small cell lung cancer (NSCLC) tissues. They indicated that MTA1 upregulation was closely related to the invasion and metastasis of NSCLC. Following that, several studies were performed to investigate the role and mechanism of MTA1 in lung cancer promotion (24-28). However, only NSCLC tissues or cell lines were examined in these studies. Whether MTA1 is also upregulated and tumor-promoting in SCLC is still unexplored to date. To resolve this question, we first detected the expression of MTA1 in SCLC by tissue microarray and the results showed that, compared to the normal lung tissues, MTA1 was significantly increased in SCLC, raising a probable role of MTA1 in SCLC promotion.

Invasion and metastasis are typical hallmarks of tumors. They are the main causes that makes it difficult to eradicate all tumor cells from the body. Although the role of MTA1 in cancer metastasis is strongly indicated in a wide range of human cancers, whether MTA1 is a potential target for cancer treatment remains poorly studied, particularly in SCLC. Nicolson et al (29) found that suppression of MTA1 by antisense oligodeoxynucleotides greatly inhibited the growth and metastasis of the MDA-MB-231 cancer cell line; Qian et al (30) also found that deletion of MTA1 by RNA interference suppressed the growth and metastasis of B16F10 melanoma cells in vitro.

In the present study, we aimed to evaluate the therapeutic value of MTA1 disruption in SCLC treatment. The NCI-H446 cell line was selected because of its high expression of MTA1 among the screened cell line models. Transfection of siRNA specifically and significantly downregulated the MTA1 expression in NCI-H446 cells, while there were no significant changes in the NC-siRNA and mock groups, demonstrating the high specificity and efficacy of the MTA1 siRNA. After MTA1 was downregulated by MTA1 siRNA, the cell growth and colony formation of NCI-H446 cells were significantly decreased, which was consistent with the properties in previous reports $(29,31)$, indicating that MTA1 mediated the regulation of the growth and proliferation of SCLC NCI-H446 cells in vitro. The adhesion and invasion of NCI-H446 cells were also significantly impaired by downregulation of MTA1. MTA1 is a downstream effector of transforming growth factor- $\beta 1$ (TGF- $\beta 1$ ) which mediates repression of epithelial-cadherin (E-cadherin) expression (32). E-cadherin is essential for maintaining cell-cell junctions which are important for tumor cell invasion and metastasis (33). Disruption of E-cadherin is correlated with tumor progression, invasive growth, metastasis and poor prognosis (34). Notably, upregulation of E-cadherin was observed after MTA1 downregulation, which coincided with the adhesion and invasion results. Cell migration ability was corroborated by a wound healing assay, and the results were further confirmed. Collectively, these findings indicate that inhibition of MTA1 significantly decreased the invasion 
and metastasis of SCLC in vitro, showing promise as a target in cancer treatment.

The MTA1/NuRD complex as a transcriptional co-repressor of tumor suppressors is associated with deacetylated p53 $(35,36)$. p53 was downregulated in the SCLC cell line following MTA1 overexpression, while depletion of MTA1 caused significant apoptosis as detected by flow cytometry and western blotting for cleaved PARP, which is possibly associated with upregulation of PUMA. Moreover, MTA1 also plays a crucial role in the DNA repair process (37). MTA1 is involved in DNA damage repair through a variety of ways, among which the poly(ADP-ribose) polymerase (PARP)-dependent manner was emphasized in human cancer cells (38). It was reported that overexpression of MTA1 could lead to the activation of oncogenic cyclin D1 pathways (39), but no significant change was observed when MTA1 was downregulated. This may be due to the complex process as MTA1 has different regulatory effects on all cycle-related proteins in different cancers. In summary, our results showed that downregulation of MTA1 significantly reduced the tumor malignant phenotype of SCLC in vitro, guaranteeing further exploration of its application in SCLC treatment.

Our research collectively indicates that MTA1 is a key regulator in SCLC. Reduction in MTA1 is an effective gene therapeutic method by which to treat SCLC in vitro by decreasing proliferation, invasion and metastasis abilities and stimulating the apoptosis pathway. This may result in the development of new treatments for SCLC.

\section{Acknowledgements}

The present study was supported by grants from the National Natural Science Foundation of China (81372158, 81372159 and 81101518).

\section{References}

1. Parkin DM, Bray F, Ferlay J and Pisani P: Global cancer statistics, 2002. CA Cancer J Clin 55: 74-108, 2005.

2. Owonikoko TK, Ragin CC, Belani CP, Oton AB, Gooding WE, Taioli E and Ramalingam SS: Lung cancer in elderly patients: an analysis of the surveillance, epidemiology, and end results database. J Clin Oncol 25: 5570-5577, 2007.

3. Jackman DM and Johnson BE: Small-cell lung cancer. Lancet 366: 1385-1396, 2005.

4. Le Péchoux C, Dunant A, Senan S, Wolfson A, Quoix E and Faivre-Finn C: Standard-dose versus higher-dose prophylactic cranial irradiation (PCI) in patients with limited-stage smallcell lung cancer in complete remission after chemotherapy and thoracic radiotherapy (PCI 99-01, EORTC 22003-08004, RTOG 0212, and IFCT 99-01): a randomised clinical trial. Lancet Oncol 10: 467-474, 2009.

5. Asai N, Ohkuni Y, Kaneko N, Yamaguchi E and Kubo A: Relapsed small cell lung cancer: treatment options and latest developments. Ther Adv Med Oncol 6: 69-82, 2014.

6. Hanahan D and Weinberg RA: Hallmarks of cancer: the next generation. Cell 144: 646-674, 2011.

7. Kumar R, Wang RA and Bagheri-Yarmand R: Emerging roles of MTA family members in human cancers. Semin Oncol 30: 30-37, 2003.

8. Xue Y, Wong J, Moreno GT, Young MK, Côté J and Wang W: NURD, a novel complex with both ATP-dependent chromatinremodeling and histone deacetylase activities. Mol Cell 2: 851-861, 1998.

9. Jang KS, Paik SS, Chung H, Oh YH and Kong G: MTA1 overexpression correlates significantly with tumor grade and angiogenesis in human breast cancers. Cancer Sci 97: 374-379, 2006.
10. Li SH, Wang Z and Liu XY: Metastasis-associated protein 1 (MTA1) overexpression is closely associated with shorter disease-free interval after complete resection of histologically node-negative esophageal cancer. World J Surg 33: 1876-1881, 2009.

11. Kawasaki G, Yanamoto S, Yoshitomi I, Yamada S and Mizuno A: Overexpression of metastasis-associated MTA1 in oral squamous cell carcinomas: correlation with metastasis and invasion. Int J Oral Maxillofac Surg 37: 1039-1046, 2008.

12. Higashijima J, Kurita N, Miyatani T, Yoshikawa K, Morimoto S, Nishioka M, Iwata T and Shimada M: Expression of histone deacetylase 1 and metastasis-associated protein 1 as prognostic factors in colon cancer. Oncol Rep 26: 343-348, 2011.

13. Zhu X, Guo Y, Li X, Ding Y and Chen L: Metastasis-associated protein 1 nuclear expression is associated with tumor progression and clinical outcome in patients with non-small cell lung cancer. J Thorac Oncol 5: 1159-1166, 2010.

14. Li DQ, Pakala SB, Nair SS, Eswaran J and Kumar R: Metastasis-associated protein 1/nucleosome remodeling and histone deacetylase complex in cancer. Cancer Res 72: 387-394, 2012.

15. Tian LL, Yue W, Zhu F, Li S and Li W: Human mesenchymal stem cells play a dual role on tumor cell growth in vitro and in vivo. J Cell Physiol 226: 1860-1867, 2011.

16. Qian H, Lu N, Xue L, Liang X, Zhang X, Fu M, et al: Reduced MTA1 expression by RNAi inhibits in vitro invasion and migration of esophageal squamous cell carcinoma cell line. Clin Exp Metastasis 22: 653-662, 2005.

17. Wang $\mathrm{H}$, Zhang $\mathrm{D}$, Wu W, Zhang J, Guo D, Wang Q, et al: Overexpression and gender-specific differences of SRC-3 (SRC-3/AIB1) immunoreactivity in human non-small cell lung cancer: an in vivo study. J Histochem Cytochem 58: 1121-1127, 2010.

18. Rudin CM, Durinck S, Stawiski EW, Poirier JT, Modrusan Z, Shames DS, et al: Comprehensive genomic analysis identifies SOX2 as a frequently amplified gene in small-cell lung cancer. Nat Genet 44: 1111-1116, 2012.

19. Sidransky D: Emerging molecular markers of cancer. Nat Rev Cancer 2: 210-219, 2002.

20. Toh Y, Pencil SD and Nicolson GL: A novel candidate metastasis-associated gene, mtal, differentially expressed in highly metastatic mammary adenocarcinoma cell lines. cDNA cloning, expression, and protein analyses. J Biol Chem 269: 22958-22963, 1994.

21. Toh Y, Oki E, Oda S, Tokunaga E, Ohno S, Maehara Y, Nicolson GL and Sugimachi K: Overexpression of the MTA1 gene in gastrointestinal carcinomas: correlation with invasion and metastasis. Int J Cancer 74: 459-463, 1997.

22. Toh Y and Nicolson GL: The role of the MTA family and their encoded proteins in human cancers: molecular functions and clinical implications. Clin Exp Metastasis 26: 215-227, 2009.

23. Sasaki H, Moriyama S, Nakashima Y, Kobayashi Y, Yukiue H, Kaji M, Fukai I, Kiriyama M, Yamakawa Y and Fujii Y: Expression of the MTA1 mRNA in advanced lung cancer. Lung Cancer 35: 149-154, 2002.

24. Xu L, Mao XY, Fan CF and Zheng HC: MTA1 expression correlates significantly with cigarette smoke in non-small cell lung cancer. Virchows Arch 459: 415-422, 2011.

25. Zhu X, Zhang X, Wang H, Song Q, Zhang G, Yang L, et al: MTA1 gene silencing inhibits invasion and alters the microRNA expression profile of human lung cancer cells. Oncol Rep 28: 218-224, 2012.

26. Xia Y, Chen Q, Zhong Z, Xu C, Wu C, Liu B and Chen Y: Down-regulation of miR-30c promotes the invasion of non-small cell lung cancer by targeting MTA1. Cell Physiol Biochem 32: 476-485, 2012

27. Li S, Tian H, Yue W, Li L, Gao C, Si L, Li W, Hu W, Qi L and $\mathrm{Lu}$ M: Down-regulation of MTA1 protein leads to the inhibition of migration, invasion, and angiogenesis of non-small-cell lung cancer cell line. Acta Biochim Biophys Sin (Shanghai) 45: 115-122, 2013.

28. Li Y, Chao Y, Fang Y, Wang J, Wang M , Zhang H, Ying M, Zhu $X$ and Wang H: MTA1 promotes the invasion and migration of non-small cell lung cancer cells by downregulating miR-125b. J Exp Clin Cancer Res 32-33, 2003.

29. Nicolson GL, Nawa A, Toh Y, Taniguchi S, Nishimori K and Moustafa A: Tumor metastasis-associated human MTA1 gene and its MTA1 protein product: role in epithelial cancer cell invasion, proliferation and nuclear regulation. Clin Exp Metastasis 20: 19-24, 2003 
30. Qian H, Yu J, Li Y, Wang H, Song C, Zhang X, Liang X, Fu M and Lin C: RNA interference of metastasis-associated gene 1 inhibits metastasis of B16F10 melanoma cells in a C57BL/6 mouse model. Biol Cell 99: 573-581, 2007.

31. Zhang H, Stephens LC and Kumar R: Metastasis tumor antigen family proteins during breast cancer progression and metastasis in a reliable mouse model for human breast cancer. Clin Cancer Res 12: 1479-1486, 2006.

32. Pakala SB, Singh K, Reddy SD, Ohshiro K, Li DQ, Mishra L and Kumar R: TGF- $\beta 1$ signaling targets metastasis-associated protein 1 , a new effector in epithelial cells. Oncogene 30: 2230-2241, 2011.

33. Thiery JP: Epithelial-mesenchymal transitions in tumour progression. Nat Rev Cancer 2: 442-454, 2002.

34. Kang Y and Massagué J: Epithelial-mesenchymal transitions: twist in development and metastasis. Cell 118: 277-279, 2004.

35. Moon HE, Cheon $\mathrm{H}$ and Lee MS: Metastasis-associated protein 1 inhibits p53-induced apoptosis. Oncol Rep 18: 1311-1314, 2007.
36. Kai L, Samuel SK and Levenson AS: Resveratrol enhances p53 acetylation and apoptosis in prostate cancer by inhibiting MTA1/ NuRD complex. Int J Cancer 126: 1538-1548, 2010.

37. Schmidt DR and Schreiber SL: Molecular association between ATR and two components of the nucleosome remodeling and deacetylating complex, HDAC2 and CHD4. Biochemistry 38: 14711-1477, 1999.

38. Chou DM, Adamson B, Dephoure NE, Tan X, Nottke AC, Hurov KE, Gygi SP, Colaiácovo MP and Elledge SJ: A chromatin localization screen reveals poly (ADP ribose)-regulated recruitment of the repressive polycomb and NuRD complexes to sites of DNA damage. Proc Natl Acad Sci USA 107: 18475-1880, 2010.

39. Bagheri-Yarmand R, Talukder AH, Wang RA, Vadlamudi RK and Kumar R: Metastasis-associated protein 1 deregulation causes inappropriate mammary gland development and tumorigenesis. Development 131: 3469-3479, 2004. 